\title{
Incentivos para la participación activa del alumnado: experiencia en materias de contabilidad
}

\author{
Rodríguez-Gulías, María Jesús; Anido-Martínez, Cristina'; Muíño, Flora
}

Universidade da Coruña. Facultade de Economía e Empresa

\section{RESUMEN}

El trabajo describe la puesta en práctica de un enfoque para la docencia en materias de contabilidad orientado a convertir al estudiante en un participante activo en el proceso de aprendizaje. El objetivo último es que el alumnado pueda continuar su formación, ya de manera autónoma, al abandonar la universidad, lo que le permitirá adaptarse a los continuos cambios a los que deberá enfrentarse en el ejercicio de su profesión. Con este fin, en la metodología docente se promueve el aprender haciendo; el papel del profesorado deja de ser el de proveer de conocimiento, para pasar a apoyar, orientar y estimular al estudiante para que derive, por sí mismo, la solución a las problemáticas objeto de estudio en la asignatura. El trabajo en clase se completa con un importante volumen de tareas a realizar fuera del aula. En coherencia con la metodología docente, el sistema de evaluación contempla, a través de diferentes actividades de evaluación continua, el esfuerzo realizado por los estudiantes en clase, pero, fundamentalmente, el trabajo llevado a cabo fuera del aula. Con las actividades propuestas se persigue, además, el desarrollo de habilidades interpersonales y de comunicación, esenciales para nuestros egresados en su ejercicio profesional.

PALABRAS CLAVE: aprendizaje autónomo, aprender haciendo, evaluación continua. 


\section{CITA RECOMENDADA:}

Rodríguez-Gulías, M. J., Anido-Martínez, C., Muíño, F.(2018). Incentivos para la participación activa del alumnado: experiencia en materias de contabilidad. En E. de la Torre Fernández (ed.) (2018). Contextos universitarios transformadores: retos e ideas innovadoras. II Xornadas de Innovación Docente. Cufie. Universidade da Coruña (pág 111-123).

DOl capítulo: https://doi.org/10.17979/spudc.9788497496780.111

DOI libro: https://doi.org/10.17979/spudc. 9788497496780

\section{ABSTRACT}

The paper describes the implementation of a teaching approach in accounting subjects aimed at promoting the active involvement of students in the learning process. The ultimate goal is that students can continue their training, in an autonomous way, upon leaving university, which will allow them to adapt to the continuous changes they will have to face in the exercise of their profession. To this end, the teaching methodology promotes learning by doing; the role of the teacher ceases to be that of providing knowledge, to support, guide and encourage students to derive, by themselves, the solution to the problems addressed in the subject. The work in class is completed with an important volume of tasks to be done outside the classroom. In coherence with the teaching methodology, the evaluation system contemplates, by means of different continuous assessment activities, the effort made by students in class, but, fundamentally, the work developed outside the classroom. With the proposed activities we also pursue the development of interpersonal and communication skills, essential for our graduates in their professional practice.

KEY WORDS: self-directed learning, learning by doing, continuous assessment. 


\section{INTRODUCCIÓN}

El objeto del presente trabajo consiste en describir la puesta en práctica, en materias introductorias de contabilidad, de un enfoque docente orientado a promover la autonomía del estudiante en el proceso de aprendizaje. La implantación de este enfoque deriva de la necesidad de incentivar al alumnado a participar activamente en el proceso formativo, de modo que pueda adquirir las competencias y habilidades necesarias para el desarrollo de la profesión en el entorno actual.

Como indica el International Accounting Education Standards Board (IAESB) (2017), el entorno cambiante en el que desarrolla su actividad el experto contable requiere profesionales con capacidad de adaptación y aprendizaje continuo. Así, la enseñanza de la contabilidad debe ir más allá de la orientación tradicional, centrada en la transferencia de conocimientos técnicos (conceptos, principios, normas o procedimientos), para establecer un marco global que considere, además, el desarrollo de habilidades intelectuales, interpersonales y de comunicación, así como de valores, ética y actitudes profesionales (IAESB, 2017, pág. 9). A este respecto, el American Institute of Certified Public Accountants (AICPA, 2017) en el AICPA Pre-Certification Core Competency Framework, defiende un currículum basado en el desarrollo de habilidades, en lugar de centrarse en las tradicionales materias 0 áreas de contenido. Entiende este organismo el aprendizaje como un proceso continuo, que comienza en el ámbito académico, pero que se extiende a lo largo de toda la vida profesional. Aunque el conocimiento necesario cambiará con el tiempo, las competencias adquiridas favorecerán el desarrollo profesional del contable en el largo plazo. En la misma línea se pronuncia Diamond (2005), quien predice que en el siglo XXI la formación contable en los grados se centrará en proporcionar a los estudiantes habilidades de aprendizaje a lo largo de toda la vida que les permitan autoformarse una vez que abandonan el ámbito académico.

Es precisamente esta orientación la que se pretende incorporar a las materias contables a través de la puesta en práctica de la metodología docente y el sistema de evaluación que se describen en este trabajo. 


\section{EL CONTEXTO}

La experiencia que se presenta se refiere a materias introductorias de contabilidad de seis ECTS impartidas en la Facultad de Economía y Empresa de la Universidade da Coruña. En este centro, una asignatura de seis ECTS lleva asociada una carga de trabajo total para el alumnado de 150 horas, de las cuales 46 se corresponden con horas de clase y 104 con trabajo autónomo. Quiere esto decir que se fomenta la autonomía de los estudiantes en el proceso de aprendizaje, ya que alrededor del $70 \%$ del trabajo deberá ser realizado fuera del aula. Esta característica de la organización docente resulta particularmente interesante para las materias de contabilidad dado que, como se ha señalado previamente, en su ejercicio profesional, los futuros egresados deberán enfrentarse a un entorno sujeto a continuos cambios, lo que les obligará a desarrollar y mantener su competencia profesional a lo largo de toda su carrera. En esta línea, en la introducción a sus pronunciamientos, el IAESB (2017) destaca, que en el contexto cambiante en el que el profesional de la contabilidad debe desarrollar su trabajo, el aprendizaje continuado es esencial y le obligará a buscar activamente la formación, práctica, conocimiento y destrezas que necesite para anticiparse y adaptarse a los cambios en procesos, tecnología, normativa, requerimientos legales, demandas del empleador, etc.

De lo anterior se deduce que el objetivo fundamental de la docencia en contabilidad no es que el alumnado adquiera un elevado volumen de conocimientos, sino que desarrolle las habilidades adecuadas para buscar y aplicar el conocimiento que necesite en el momento en que lo precise. A este respecto, Kelly et al. (1999, pág. 325) apuntan que lo más importante en el proceso de educación es que el estudiante se convierta en un aprendiz independiente, que sea capaz de afrontar nuevos retos no programados, ni estructurados.

El desafío para los docentes de materias contables reside, precisamente, en conseguir que los estudiantes lleguen a convertirse en sujetos activos del proceso de aprendizaje, de modo que puedan desarrollar elevados niveles de autonomía en su formación. En nuestra opinión esto exige enfocar la materia de forma que sean los propios estudiantes los que deriven la solución a los problemas y casos abordados en la asignatura. Quiere esto decir que la docencia, en 
lugar de apoyarse en la explicación del docente, ha de orientarse al planteamiento a los estudiantes de problemas o casos, en la medida de lo posible reales 0 que simulen la realidad, para que sean los propios estudiantes los que, guiados por el profesorado, desarrollen la solución para los casos propuestos. En definitiva, lo que se persigue es que el alumnado aprenda haciendo, antes que escuchando y, con este fin, se invierte el proceso tradicional de la docencia en contabilidad; en lugar de que el docente explique una norma 0 práctica contable, para añadir después ejemplos que clarifiquen la exposición realizada, plantea un problema de partida, orienta a los estudiantes para que lo resuelvan siguiendo un proceso lógico y, finalmente, contrasta la solución con la práctica contable o norma en cuestión.

La puesta en práctica de este enfoque requiere la implicación del alumnado para mantener una constancia en el trabajo a lo largo de todo el cuatrimestre plasmada tanto en la labor realizada en clase, como en la desarrollada de manera autónoma fuera del aula. La metodología docente y el sistema de evaluación resultan esenciales para la consecución de este objetivo.

\section{METODOLOGÍA DOCENTE}

La aplicación del enfoque anteriormente mencionado requiere la utilización de una metodología docente en la que el estudiante sea el centro de atención. En particular, los elementos que distinguen la metodología empleada en la asignatura a la que se refiere esta experiencia docente son los siguientes:

- Planificación del trabajo semanal

- Diseño de las tareas

- Apoyo de las TIC

- Papel del profesorado

\subsection{Planificación del trabajo semanal}

Las materias a las que se refiere esta experiencia tienen asignadas tres horas de docencia presencial a la semana, repartidas en dos sesiones de 90 minutos. Teniendo esto en cuenta, el 
contenido del curso se ha estructurado en 12 capítulos, de forma que se destine una semana a cada tema. Las dos semanas restantes se dedicarán, una a la presentación del trabajo de curso y otra a recapitulación.

Dentro de esta planificación semanal, en la primera sesión se plantean problemas y casos básicos que el alumnado debe resolver en el aula con las pertinentes orientaciones por parte del profesorado. Al término de esta sesión los estudiantes deberían haber desarrollado el conocimiento y las habilidades necesarias para solucionar, por su cuenta (fuera del aula), los casos prácticos que acompañan al tema. La segunda sesión se dedica, precisamente, al comentario y discusión de los mencionados casos prácticos. Como se explicará en el apartado destinado a la evaluación, esta segunda sesión comienza con una mini-prueba escrita, similar a los ejercicios propuestos para resolver fuera del aula, pero más simple, destinada a evaluar si el alumnado ha realizado las tareas propuestas.

Esta planificación semanal facilita la organización de la docencia, al distribuir el contenido de manera uniforme a lo largo del cuatrimestre, y resulta también muy útil desde un punto de vista pedagógico, porque en cada sesión se aborda un capítulo y se termina con una conclusión o reflexión global que ayuda a asentar el conocimiento adquirido. Además, con esta programación las tareas que se proponen a los estudiantes tienen una periodicidad semanal, lo que introduce un cierto hábito o disciplina de trabajo.

\subsection{Diseño de las tareas}

Como se ha indicado previamente, el $70 \%$ del trabajo para la materia debe ser desarrollado por los estudiantes fuera del aula. El paquete de casos prácticos que acompaña a cada tema tiene por objetivo proporcionar al estudiante una guía sobre los aspectos en los que debería profundizar con respecto al tema en cuestión.

Debido a la relevancia de los casos prácticos dentro del enfoque dado a la materia, éstos se diseñan con meticulosidad. En primer lugar, procuramos utilizar casos de empresas reales (utilizando, por ejemplo, los informes publicados por las empresas) o problemas que simulen 
la realidad. Cuando se busca la participación activa de los estudiantes en las clases, la utilización de ejemplos reales o que se aproximen a la realidad es muy útil para atraer su atención. Además, les ayuda a recodar cómo resolver un determinado problema, porque les resulta más sencillo visualizar mentalmente el caso en cuestión y el proceso seguido para su resolución. Por último, es una buena vía para que los estudiantes se acerquen al ejercicio de la profesióni.

En segundo lugar, proponemos casos con diferentes niveles de dificultad. Los supuestos más sencillos pretenden clarificar aspectos esenciales y animar al alumnado en la resolución de las tareas. Los de nivel intermedio tienen como objetivo afianzar conocimientos y destrezas, mientras que los más difíciles se presentan a modo de reto para los estudiantes con mayor predisposición a profundizar en los temas analizados.

Por último, siempre que es posible, el enunciado del caso práctico ofrece un dato de comprobación, muy apreciado por los estudiantes porque les permite verificar si la solución que han derivado es la correcta.

\subsection{Apoyo de las TIC}

En la actualidad, las tecnologías de la información y la comunicación no sólo representan una herramienta atractiva para el alumnado, sino también son fundamentales para su desempeño profesional futuro y favorecen su posterior incorporación al mercado laboral. Esta es la razón por la que en el desarrollo de esta materia se contempla la utilización, entre otras, de las siguientes herramientas: software de contabilidad, hoja de cálculo, herramientas de presentación y foro virtual.

El software de contabilidad es especialmente interesante por su conexión directa con el ámbito laboral. Por otra parte, permite dar relevancia a los contenidos de la materia frente a la carga numérica y mecánica propias de la asignatura.

Las herramientas de ofimática optimizan ciertas tareas. Así, en el bloque destinado al análisis e interpretación de los estados financieros, la hoja de cálculo agiliza el tratamiento de los datos y 
su representación gráfica, mientras que el programa de presentación favorece la transmisión de los resultados a los oyentes de la exposición.

Por último, dentro de la plataforma Moodle, hemos creado un foro virtual para el planteamiento y la resolución de dudas por el propio alumnado. Es práctica habitual entre los estudiantes crear grupos virtuales. En este caso, un aliciente es que el docente es el moderador y, por lo tanto, puede validar las respuestas ofrecidas u orientar para la correcta resolución de la duda.

\subsection{Papel del profesorado}

De la descripción realizada en los epígrafes anteriores se deduce que el proceso de aprendizaje se centra en el recorrido que realiza el estudiante hasta alcanzar la solución a los problemas analizados. Durante el camino, surgen dudas, se cometen errores, se entablan debates, potenciando todo ello la adquisición de conocimientos técnicos, así como el desarrollo de capacidades interpersonales y de comunicación. El papel protagonista recae en el discente y, en consecuencia, el docente actúa como un guía que cumple las misiones de orientar, apoyar, promover 0 estimular el trabajo de los estudiantes.

Siguiendo la máxima de aprender haciendo, el profesorado plantea las cuestiones contables a analizar, así como los conceptos básicos a tener en cuenta. Entonces, el alumnado no se limita a escuchar, debe participar activamente en la elaboración del conocimiento. El docente, en su faceta de orientador, guía al estudiante en la búsqueda de la solución. Este contacto directo permite identificar aptitudes y dificultades individuales. Posibilita así la prestación de un apoyo personalizado, tanto para mantener el interés de quienes avanzan con mayor facilidad, planteándoles retos adicionales, como para evitar la desmoralización de quienes se enfrentan a un proceso de aprendizaje más lento, ofreciéndoles orientaciones ajustadas a sus necesidades.

Las dudas surgidas o los errores cometidos durante el proceso son objeto de debate. Es responsabilidad del profesorado estimular la participación y el trabajo de todo el alumnado. 


\section{SISTEMA DE EVALUACIÓN}

En coherencia con el objetivo propuesto y la metodología docente utilizada, el sistema de evaluación contempla diferentes elementos destinados a promover la constancia en el trabajo y la autonomía de los estudiantes en el proceso de aprendizaje. Como señala Gibbs (2007), el sistema de evaluación es la herramienta más poderosa de la que dispone el profesorado para influir en la actitud y el trabajo desarrollado por los estudiantes. Esta es la razón por la que, en nuestro caso, la labor de los estudiantes, particularmente aquella realizada de manera autónoma (fuera del aula), alcanza una elevada ponderación en el cómputo de la calificación. El examen final representa el $40 \%$ de la nota en el acta; el resto corresponde a la evaluación continua, la cual incluye los siguientes elementos:

\section{- Mini-pruebas prácticas}

Se realizan al inicio de cada una de las sesiones destinadas a discutir los casos prácticos y están diseñadas para evaluar si el alumnado ha realizado las tareas encomendadas, en cuyo caso no suponen dificultad alguna. De las pruebas realizadas, generalmente 12 , se valoran únicamente cuatro, elegidas aleatoriamente al finalizar el cuatrimestre. De esta manera, se pretende asegurar un desempeño uniforme a lo largo del tiempo.

\section{- Presentación del trabajo de curso}

Dentro del marco de los temas dedicados a la interpretación de los estados financieros, se propone la elaboración y presentación en el aula de un trabajo que versará sobre una empresa real y que será desarrollado en grupos de tres 0 cuatro estudiantes.

En la valoración de esta tarea, se tiene en cuenta el rigor en su resolución, pero también la claridad en la exposición y la capacidad para responder a las cuestiones planteadas por profesorado y alumnado, así como la habilidad para trabajar en equipo. Se pretende con ello contribuir al desarrollo de habilidades transversales como las interpersonales y de comunicación, esenciales para el ejercicio profesional de 
nuestros futuros egresados (Arquero Montaño et al., 2008); Gómez-Ruiz y NaranjoGil, 2011).

\section{- Participación en clase}

Se valora la participación activa en el desarrollo de la materia, tanto en el aula, como virtualmente, en el foro habilitado para el planteamiento y resolución de dudas.

\section{- Test intermedio}

Se trata de una prueba tipo test realizada al terminar el primer bloque de la materia que permite a los estudiantes validar su evolución y, en el caso de ser necesario, tomar medidas correctivas.

Para el cómputo de la nota correspondiente a la evaluación continua, cada uno de los siete ítems anteriores (4 mini-pruebas, trabajo, participación y test) representa un $10 \%$ de la calificación final; una vez realizados los cálculos se eligen las seis mejores notas. Se pretende con ello evitar el arbitrio de soluciones alternativas, siempre cuestionables en la evaluación continua, para aquellos casos en los que el estudiante no pueda estar presente en alguna de las pruebas. Esta holgura reduce, asimismo, el estrés del alumnado cuando intuye que el resultado de alguna de las evaluaciones no es satisfactorio.

\section{RESULTADOS}

Nuestra experiencia con la utilización de esta orientación en materias introductorias de contabilidad se inicia en el curso 2011/12 y, aunque en los primeros años las tareas semanales y las correspondientes mini-pruebas generaban un cierto rechazo entre el alumnado, con el paso de los cursos, esta metodología y sistema de evaluación se han ido asentando y creemos que, en estos momentos, los estudiantes son plenamente conscientes de que este enfoque les resulta beneficioso. Intuimos que la experiencia de alumnos de promociones anteriores les sirve de orientación y les ayuda a comprender cuál puede ser la recompensa a la distribución del esfuerzo a lo largo de todo el cuatrimestre. 
Aunque existen diferencias apreciables entre títulos y promociones, consideramos que la utilización de esta metodología y sistema de evaluación favorece al alumnado, entre otros, en los siguientes aspectos:

- La tasa de evaluación (presentados/matriculados) tiende a ser elevada (superior al 90\%). Conseguir que la mayor parte del alumnado participe en las actividades de evaluación continua y, por tanto, mantenga un nivel razonable de esfuerzo a lo largo del cuatrimestre, ha sido una importante preocupación para el equipo docente de la materia. La razón es que el rendimiento en asignaturas contables suele ser muy reducido cuando el trabajo se concentra en los días previos al examen final.

- Las calificaciones finales suelen ser altas, con un elevado porcentaje de notas superior al siete, si bien la tasa de rendimiento (aprobados/matriculados) es, generalmente, moderada. Los suspensos corresponden a estudiantes que participan esporádicamente en las actividades de evaluación continua. Su rendimiento es pobre no sólo en estas tareas, sino también en el examen final. En una materia como esta, en la que para comprender un determinado tema es necesario dominar los anteriores, la constancia en el trabajo es esencial.

- Se fomenta la autonomía de los estudiantes en el proceso de aprendizaje y éstos llegan a ser conscientes de su capacidad para resolver problemas que no les son familiares.

- Se contribuye al desarrollo de habilidades transversales, tales como las interpersonales y de comunicación. Con el avance del curso se muestran menos reacios a participar en (o incluso iniciar) las discusiones promovidas en el aula y se esfuerzan en proporcionar argumentos con los que defender su punto de vista.

Todo lo anterior promueve un cambio en la actitud del alumnado hacia la materia; el rechazo inicial hacia la misma, propio de las asignaturas cuantitativas, va desapareciendo a medida que avanza el curso y comprueban que se trata de una disciplina que resulta asequible si se acompaña de un nivel razonable de perseverancia en el trabajo. 
Por último, hemos que de mencionar que los beneficios de la utilización de este enfoque alcanzan también a los docentes. En primer lugar, las clases son participativas y esto las hace diferentes en cada grupo. Se reduce, de esta forma, el tedio que puede suponer para el profesorado impartir clase de la misma materia en varios grupos. En segundo lugar, la participación activa de los alumnos en el aula y su perseverancia en el trabajo permiten al docente identificar los problemas a los que se enfrenta cada uno de ellos y ofrecerles apoyo personalizado. Por último, el número de estudiantes repetidores y desmotivados tiende a reducirse, y esto repercute positivamente en la implicación del resto del alumnado en el trabajo semanal.

\section{CONCLUSIONES}

La experiencia que aquí se describe se orienta a convertir al estudiante en un participante activo en el proceso de aprendizaje, de modo que pueda continuar su formación, ya de manera autónoma, al abandonar la universidad. Esto le permitirá adaptarse a los continuos cambios a los que deberá enfrentarse en el ejercicio de su profesión.

Aun cuando la metodología docente y el sistema de evaluación utilizados fomentan la disciplina en el trabajo y la autonomía en el aprendizaje, somos conscientes de que hay todavía un margen considerable para la mejora. Así, persiste un porcentaje de estudiantes, en algunos casos no desdeñable, que no participa en las actividades de evaluación continua propuestas o que no es constante en el desarrollo de estas tareas. Reducir este porcentaje contribuiría a mejorar las tasas de rendimiento, a la vez que facilitaría el trabajo en el aula, dado que el grado de avance de los diferentes estudiantes sería más homogéneo. Para tratar de aliviar este problema introdujimos, en el curso 2015/16, el trabajo a presentar en el aula, como una actividad adicional de evaluación continua y, en el curso 2016/17, el foro de Moodle, a modo de refuerzo de las tutorías. Dado que ambas experiencias han sido positivas, pretendemos profundizar en estas vías u otras equivalentes con la finalidad de maximizar la participación activa del alumnado en el proceso de aprendizaje. 


\section{REFERENCIAS}

- Arquero Montaño, J.L, Jiménez Cardoso, S. M. y Joyce, J. (2004). Skills development, motivation and learning in financial statement analysis: an evaluation of alternative types of case studies. Accounting Education, 13 (2), pp. 191-212.

- American Institute of Certified Public Accountants (AICPA) (2017): AICPA PreCertification Core Competency Framework, disponible en

- https://www.aicpa.org/interestareas/accountingeducation/resources/corecompetency. html, fecha de la última descarga: 23/11/2017.

- Diamond, M. (2005). Accounting Education, Research and Practice: After Enron, Where Do We Go? European Accounting Review, 14 (2), pp. 353-362.

- Gibbs, G. (2007). Uso estratégico de la evaluación en el aprendizaje. (61-70). En S.A. Brown y A. Glasner (Eds.). Evaluar en la universidad. Problemas y nuevos enfoques. Madrid: Narcea.

- Gómez Ruiz, L.M. y Naranjo Gil, D. (2011). La competencia de trabajo en grupo: una propuesta de actividad y de evaluación. Revista de Docencia Universitaria, 9 (2), pp. 193-211.

- International Accounting Education Standards Board (IAESB) (2017): Handbook of International Education Pronouncements. New York, International Federation of Accountants.

- Kavanagh, M. H. y Drennan, L. (2008). What skills and attributes does an accounting graduate need? Evidence from student perceptions and employer expectations, Accounting and Finance, 48 (2), pp. 279-300.

- Kelly, M., Davey, H. y Haigh, N. (1999). Cotemporary accounting education and society. Accounting Education, 8 (4), pp. 321-340.

\footnotetext{
' Los empleadores esperan de los graduados un cierto nivel de conocimiento del mundo de los negocios 0 experiencia en la vida real (Kavanagh y Drennan, 2008). Si bien el trabajar con casos de empresas reales no se puede considerar equivalente a una experiencia profesional, sí aporta un primer contacto con la realidad empresarial.
} 